\title{
Stratégies d'échantillonnage et d'analyse appliquées à l'étude des polluants organiques dans les eaux côtières
}

\section{Sampling and analysis of organic pollutants in coastal waters}

\author{
A. Saliot, I. Bouloubassi et E. Lipiatou \\ Laboratoire de Physique et Chimie Marines de l'Université Pierre et Marie Curie, \\ UA CNRS $n^{\circ}$ 353, 4, place Jussieu, 75252 Paris Cedex 05, France
}

\begin{abstract}
Résumé. - Les hydrocarbures sont un danger majeur pour l'océan. Dans les eaux côtières ce type de contamination est exacerbé par les effets de synergie entre de multiples sources de poliution. Le développement de programmes permettant de dresser des bilans des différents apports et la nécessité de concevoir des modèles prédiclifs permeltant d'évaluer l'impact des hydrocarbures et des autres contaminants sur la qualité des eaux marines et sur les écosystèmes côtiers reposent sur la qualité des stratégies d'échantillonnage et d'analyse mises en place. Cette revue décrit certains points importants de ces stratégies, appliquées au delta du Rhône et au bassin Méditerranéen Nord-occidental. L'échantillonnage doit comprendre des prélèvements effectués aux étapes clés du fonctionnement du fleuve et des activités biologiques se développant dans les zones frontales, au large du fleuve. La stratégie d'échantillonnage comprend l'étude de la diffusion des eaux fluviales et de leurs polluants associés en milieu marin et l'étude des zones de création et d'accumulation de particules. L'échantillonnage s'intéresse à divers compartiments: eau, particules en suspension (y compris les organismes vivants), couches turbides se développant près du fond, sédiments superficiels. Après une couverture complète de paramètres clés, tels salinité, MES, Carbone organique, $\mathrm{O}_{2}, \mathrm{pH}$, une étude détaillée et quantitative sera effectuée sur les hydrocarbures par combinaison de diverses techniques chromatographiques. Les empreintes obtenues peuvent être discutées en termes d'identification de diverses sources naturelles et anthropiques, de leur disponibilité pour les organismes vivants en fonction de leur répartition entre phases dissoute et particulaire, de transport, de dégradation et d'ultime dépôt dans les sédiments. Des exemples illustrent l'intérêt des analyses d'hydrocarbures aromatiques polycycliques dans les sédiments; ils signent des empreintes fidèles des divers types de contamination que l'on peut rencontrer en milieu marin côtier.
\end{abstract}

Mots-clés. - Delta du Rhône - Hydrocarbures - Méditerranée Nord-occidentale - Pollution - Traceurs géochimiques. 


\begin{abstract}
Hydrocarbons represent a major threat to the world's oceans. This type of organic contamination is exacerbated in coastal waters by the synergetic effects of multiple sources of pollution. The development of programs to estimate the budgets of the various external elements, and the designing of predictive models to evaluate the impact of hydrocarbons and other contaminants on marine water quality and on coastal ecosystems depend on the quality of the sampling and analytical techniques used. This presentation describes certain key features of these techniques as applied to the Rhone delta and the northwestern Mediterranean basin. Sampling must be performed at key stages in the river dynamics and in the biological activities which develop in the frontal zones beyond the mouth of the river. The strategy chosen for sampling includes study of the dispersion of river waters and their associated pollutants in the marine environment, as well as study of the zones of generation and accumulation of particles. Sampling involves various compartments: water, suspended matter (including living organisms), turbid layers developing near the bottom and superficial sediment. Following determination of all key parameters such as salinity, suspended matter, organic carbon, $\mathrm{O}_{2}$ and $\mathrm{pH}$, a detailed, quantitative analysis of hydrocarbons is carried out, combining various chromatographic techniques. The resulting distribution patterns are discussed in terms of identification of various natural and anthropogenic sources, their availability for living organisms as a function of their dissolved and particulate distribution, transport, degradation and ultimate settling in sediments. Examples are given to illustrate the value of analysis in sediment samples of polycyclic aromatic hydrocarbons, which give distribution patterns that clearly reveal the various types of contamination potentially encountered in the coastal marine environment.
\end{abstract}

Key words. - Geochemical tracers - Hydrocarbons - Northwestern Mediterranean basin - Pollution - Rhône delta.

\section{INTRODUCTION}

\section{Les eaux côtières et estuariennes} sont soumises à d'importants apports de matière en provenance du continent. Ces apports revêtent diverses formes physiques, chimiques et biologiques qui conditionnent largement la qualité des eaux côtières, leur productivité ef le maintien d'activités traditionnelles telles que la pêche, l'aquaculture et, plus généralement, l'exploitation du milieu marin. Le développement des activités humaines: agriculture, industrie, concentration urbaine, contribue également à l'introduction d'apports anthropogéniques qui transitent depuis les grands bas- sins fluviaux et leurs exutoires jusqu'aux estuaires et deltas, engendrant des changements profonds dans les équilibres chimiques et biologiques des eaux côtières. La question est donc souvent posée de connaître l'impact des apports anthropogéniques en prenant en compte la synergie qui se développe entre divers types d'apports et de contaminants. Dans ce contexte, les polluants chimiques inorganiques (métaux lourds) et les polluants organiques (hydrocarbures, PCBs, phénols...) jouent un rôle privilégié.

Dans le présent article nous discutons des stratégies d'échantillonnage et d'analyse mises au point afin d'é- 
tudier l'impact de polluants organiques prioritaires, les hydrocarbures, sur les eaux côtières, en choisissant un modèle simple: celui du delta du Rhône et des eaux côtières adjacentes. La Méditerranée est un modèle adéquat pour évaluer des flux de polluants à l'échelle régionale, car elle est bien connue sur le plan hydrologique. Cette mer semi-fermée reçoit globalement des quantités importantes d'hydrocarbures: environ $0,9 \times 10^{6}$ tonnes par an (Burns et Saliot, 1986), alors que les apports à l'océan mondial sont de $3-4 \times 10^{6} t$ an (NRC, 1985). Dans ce contexte, nous avons choisi d'étudier l'impact des hydrocarbures véhiculés par le Rhône, qui draine un bassin versant industrialisé, qui est l'apport majeur en eau fluviale au bassin méditerranéen Nord-occidental avec un débit moyen annuel de $1700 \mathrm{~m}^{3} / \mathrm{s}$, et qui apporte $4-5 \times 10^{6}$ tan de matières en suspension dans ce bassin (Span, 1984).

Les grandes lignes de la stratégie d'échantillonnage que nous discutons ici sont extrapolables à d'autres types d'environnement; cependant chaque aire régionale doit être considérée avec ses propres contraintes humaines, climatiques, hydrologiques et biogéochimiques.

Dans tous les cas, il convient d'évaluer les flux apportés par diverses voies aériennes, fluviales et côtières, à partir des installations industrielles et agricoles et des centres urbains, et de connaître les différentes composantes en jeu grâce à une analyse chimique fine des différents réser- voirs: aérosols et poussières, eau, particules en suspension, organismes vivants, sédiments superficiels.

Afin d'apporter les données nécessaires à la construction de modèles prédictifs, l'échelle d'observation doit permettre de couvrir la zone géographique et ses limites, mais il faut aussi prendre en compte la variabilité saisonnière climatique et biologique et la variabilité interannuelle.

Enfin ces études doivent s'inscrire dans un contexte pluridisciplinaire pour apporter une réponse aussi complète que possible aux problèmes environnementaux.

\section{STRATÉGIES D'ÉCHANTILLONNAGE}

Plusieurs éléments sont à considérer en priorité: l'emplacement des stations, leur nombre, les profondeurs de prélèvement, les éléments à collecter et le rythme des prélèvements, tout en restant dans des limites compatibles avec la stratégie analytique choisie.

Nous ne reviendrons pas sur l'importance de la contribution de l'atmosphère, sous ses composantes gazeuse et particulaire (Saliot et Marty, 1986).

Le canevas de stations doit englober les types de milieux hydriques représentés dans l'aire étudiée: eaux douces des cours d'eaux débouchant sur le littoral, eaux saumâtres représentatives du gradient de salinité dans 
la zone de mélange de l'eau douce et de l'eau salée, chenaux de navigation, eaux marines, points singuliers où s'effectuent des apports ponctuels: rejets industriels ou urbains.

Dans tous les cas on prélèvera l'eau à plusieurs profondeurs en fonction de la stratification des eaux et d'éventuelles couches turbides ou particulièrement productives ou riches biologiquement. Le prélèvement sera fait en conditions "ultra-propres" et filtré immédiatement. Le filtrat est stocké pour éviter toute évolution ultérieure jusqu'au traitement au laboratoire; les particules en suspension sont immédiatement congelées (Saliot, 1981). Le sédiment sera également prévelé sans perturbation pour obtenir des tranches dont on déterminera ultérieurement l'époque de dépôt.

On pourra compléter utilement ces prélèvements par des analyses effectuées sur des indicateurs biologiques choisis en fonction de leur capacité de concentrer les polluants; ce sont généralement des mollusques filtreurs (huîtres, moules) qui sont choisis comme dans le programme américain "Mussel Watch" (Risebrough et al., 1983) ou en France dans le Réseau National d'Observation de la Qualité du Milieu Marin (Marchand, 1985).

Cette série de prélèvements devra être répétée un certain nombre de fois afin d'estimer les flux de contaminants et leur variabilité annuelle et interannuelle.

Pour un fleuve comme le Rhône, les contraintes sont essentiellement d'ordre hydrologique: le débit, et par conséquent l'efficacité de lessivage du bassin versant varient dans une large mesure: de 920 à $4400 \mathrm{~m}^{3} / \mathrm{s}$, avec une moyenne annuelle de $1700 \mathrm{~m}^{3} / \mathrm{s}$; on sera attentif à échantillonner en période de pluies d'automne et de printemps.

La seconde contrainte est d'ordre biologique, il faut recueillir des données pendant les périodes d'intense activité biologique, particulièrement celles représentatives de la production planctonique et de l'activité bactérienne au printemps et à l'automne.

Nous présenterons les données acquises sur les sédiments superficiels et avons retenu, dans un grand lot de carottes, deux sites deltaïques échantillonnés en hiver et en été, les stations Rh1 à l'embouchure du Rhône et Rh2 à la limite du plateau continental (fig. 1) et quatre stations du Golfe du Lion collectées par $69 \mathrm{~m}$ de fond pour GL4, $750 \mathrm{~m}$ pour GL3, $1700 \mathrm{~m}$ pour $\mathrm{GL} 2$ et $2200 \mathrm{~m}$ pour GL1 (fig. 3).

\section{STRATÉGIE D'ANALYSE}

Les hydrocarbures revêtent une grande diversité structurale, dans une large gamme de poids moléculaire, de degrés d'insaturation et d'aromaticité. En termes de contamination on s'intéressera en priorité aux composés présentant le plus grand risque: les hydrocarbures aromatiques polycycliques (HAP), dont certains sont cancérigènes et mutagènes et qui présentent une forte rémanence une 


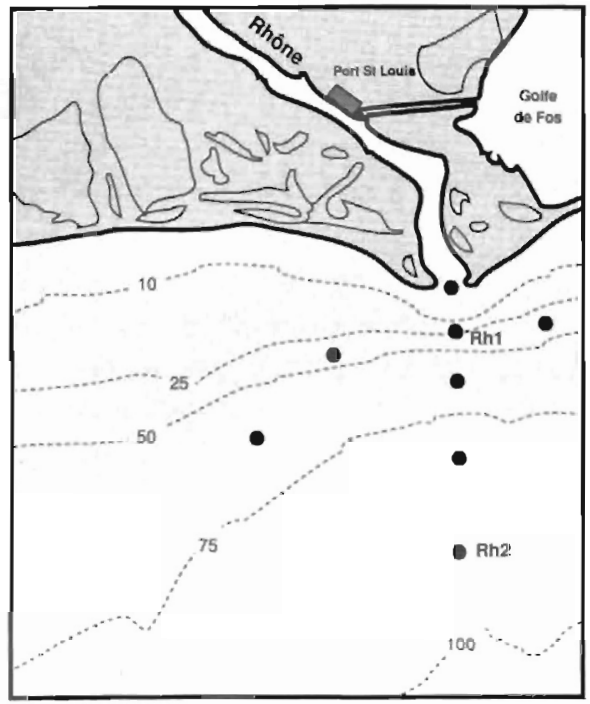

Fig. 1. - Le delta du Rhône. L'emplacement des stations de prélèvements occupées pour l'étude du delta est désignée par des ronds noirs. Les stations Rh1 et Fh2 sont discutées dans le présent article.

Fig. 1. - The Rhone delta. Location of sampling sites is showed by black dots. Stations Rh1 and Rh2 are discussed in this paper.

fois introduits dans le milieu aquatique. Toutefois une analyse détaillée de la fraction "hydrocarbures non volatils" permettra non seulement de faire un diagnostic sur les composés aromatiques, mais aussi une analyse des hydrocarbures totaux. Ceci permet d'aborder la géochimie de ces composés, de faire la part entre composés d'origines naturelle et anthropique, de suivre diverses composantes continentale, planctonique, benthique, d'identifier des composantes d'origine anthropique (pétrolière, pyrolytique industrielle, pyrolytique naturelle, feux de forêt...), d'apprécier l'état de dégradation des hydrocarbures par les processus naturels physicochimiques et/ou microbiologiques naturels ou faisant suite à une intervention humaine (dispersants, adsorbants).

Toutefois devant la complexité et le coût des analyses fines, on devra mettre en place un ensemble d'analyse plus global, par la mise en cuvre des techniques de mesure par spectrophotométrie infra-rouge ou par spectrophotométrie en UV ou par spectrofluorimétrie, qui permettent d'obtenir une cartographie à partir de nombreux prélèvements, et, ultérieurement, de sélectionner les points prioritaires nécessitant une stratégie analytique complète.

\section{EXTRACTION DES \\ HYDROCARBURES DISSOUS ET PARTICULAIRES \\ DES ÉCHANTILLONS D'EAU, DES ORGANISMES VIVANTS ET DES SÉDIMENTS}

On veillera à la propreté des conditions opératoires, particulièrement au cours des étapes réalisées à bord d'un navire de recherche ou d'un laboratoire de terrain.

L'utilisation de hottes à flux laminaire, qui permettent de travailler en conditions d'atmosphère contrôlée est recommandée. Une attention particulière doit être portée à l'utilisation d'étalons internes, en général des composés deutériés, qui n'interfèrent pas dans les séquences analytiques ultérieures et qui permettront une 
quantification rigoureuse des hydrocarbures, en s'affranchissant des différents rendements des protocoles opératoires. Ces étalons représentatifs des séries recherchées (par exemple n-C24 perdeutérié; naphthalène-D8, phénanthrène-D10; pyrèneD10; Lipiatou, 1989; Bouloubassi, 1990) seront ajoutés aux toutes premières étapes du protocole.

Il importe pour pouvoir évaluer le devenir des hydrocarbures dans des zones à forts changements physicochimiques (salinité, pH) et, par là, leur biodisponibilité, de réaliser des analyses à la fois sur les fractions dissoute, colloïdale, associée aux particules en suspension et sédimentaire. La fraction particulaire peut être séparée de l'eau par filtration sous vide modéré ou sous pression modérée, afin d'éviter l'endommagement sur le filtre des cellules vivantes fragiles (Saliot, 1981). On utilisera classiquement des filtres en fibre de verre de type Whatman GF/F, calibrés à $0,7 \mu \mathrm{m}$; ceux-ci doivent être pré-extraits par un solvant ou portés dans un four à $450{ }^{\circ} \mathrm{C}$. Toutefois il est recommandé d'utiliser des filtres de plus petite porosité, 0,1 ou $0,2 \mu \mathrm{m}$, qui permettent de retenir les microorganismes et les très petits organismes planctoniques. L'ultrafiltration devrait permettre d'obtenir la fraction colloïdale. L'extraction des hydrocarbures dissous se fait couramment par extraction liquide-liquide avec des solvants chlorés $\left(\mathrm{CH}_{2} \mathrm{Cl}_{2}, \mathrm{CHCl}_{3}\right)$, ou encore par passage de l'eau filtrée sur des colonnes remplies de mousse de polyuréthane (Delappe et al., 1983;
Albaiges et al., 1984). Le rendement obtenu par l'extraction avec des solvants chlorés, testé par Wade et Quinn (1975) est proche de $100 \%$ pour divers types d'hydrocarbures.

L'extraction des hydrocarbures associés aux particules en suspension, aux organismes vivants et aux sédiments de préférence préalablement lyophilisés peut se faire par différentes méthodes: on retiendra l'extraction en Soxhlet, avec agitation, par le chloroforme, le chlorure de méthylène ou encore un mélange de toluène et de méthanol.

\section{ISOLEMENT \\ ET FRACTIONNEMENT DES HYDROCARBURES}

L'isolement des hydrocarbures à partir des extraits lipidiques obtenus avec des solvants est une étape indispensable; il peut être réalisé par diverses techniques chromatographiques sur colonne et sur plaque (Saliot et Marty, 1986). Nous retiendrons ici la chromatographie liquide haute performance (CLHP), particulièrement élégante pour son efficacité à séparer, en une seule séquence, différentes fractions hydrocarbures, comme ceci est illustré sur la figure 2, d'après Bouloubassi ef Saliot (1991a). Brièvement la séparation présentée est effectuée grâce à une colonne $(20 \mathrm{~cm}$ de long $\times 4,8 \mathrm{~mm}$ de diamètre intérieur) remplie de silice greffée (Nucleosil-5 CN) qui permet de séparer les composés polaires. Le $n$-hexane est utilisé 


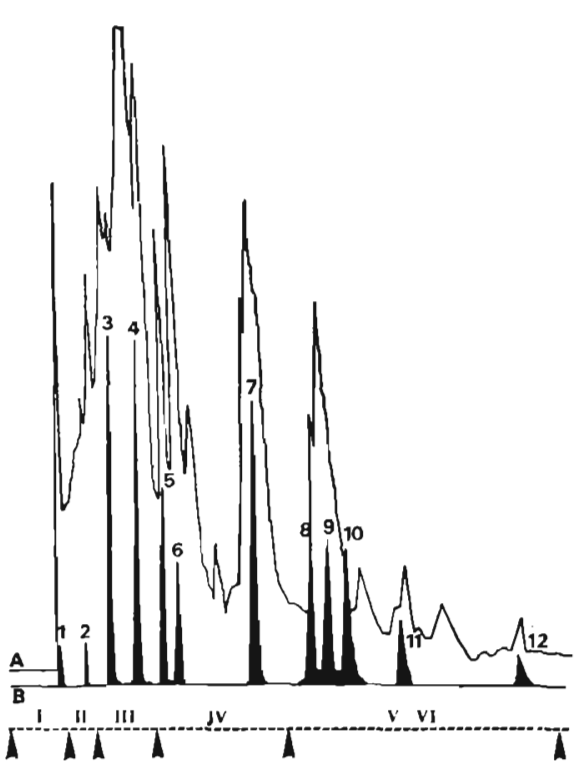

Fig. 2. - Chromatogrammes CLHP des hydrocarbures totaux. Empreinte A: sédiment superficiel prélevé à la station Rh1, près de l'embouchure du Rhòne. Empreinte B: mèlange de composés aromatiques standards: 1 : benzène, 2: naphtalène, 3 : fluorène, 4 : phénanthrène, 5 : fluoranthène, $6:$ pyrène, $7:$ chrysène, 8: benzo[b]fluoranthene, 9 : benzo[ $\%$ ]pyrène, 10: pérylène, 11: dibenzo[a,h]anthracène, 12: benzo[ghi]pérylène. Les chiffres romains désignent les fractions suivantes: I: hydrocarbures non-aromatiques et benzéniques, 11 : hydrocarbures comportant deux cycles aromatiques, III: hydrocarbures à trois cycles aromatiques, IV: hydrocarbures à 4 cycles aromatiques, V-VI: hydrocarbures à 5-6 cycles aromatiques. D'après Bouloubassi et Saliot (1991a).

Fig. 2. - HPLC chromatogrammes of total hydrocarbons. Trace A: superficial sediment at station Rh1 (river mouth). Trace B: mixture of standard compounds: 1: benzene, 2: naphthalene, 3: fluorene, 4: phenanthrene, 5: fluoranthene, 6: pyrene, 7: chrysene, 8: benzo[b]fluoranthene, 9: benzo[( $x]$ pyrene, 10: perylene, 11: dibenzo[a,h]anthracene, 12: benzo[ghi]perylene. Roman numbers refer to following fractions: 1: non-aromatic and benzenic hydrocarbons, II: PAH with 2 aromatic cycles, III: PAH with 3 aromatic cycles, IV: PAH with 4 aromatic cycles, V-VI: PAH with 5-6 aromatic cycles. After Bouloubassi and Saliot (1991a). comme phase mobile à un débit de $1,2 \mathrm{ml} / \mathrm{min}$. L'élution est suivie par spectrophotométrie en UV, à $254 \mathrm{~nm}$. En phase normale en utilisant un greffage $-\mathrm{NH} 2$ ou $-\mathrm{CN}$, la rétention des hydrocarbures croît avec le nombre de cycles aromatiques. La présence de groupes alkyles sur les structures aromatiques a peu d'effet sur le temps de rétention. Cette séquence par CLHP permet ainsi, comme le montre la figure 2, la séparation des hydrocarbures non-aromatiques et des composés aromatiques par degré d'aromaticité.

\section{ANALYSE DES HYDROCARBURES}

Rappelons brièvement les techniques dites "globales" d'évaluation des hydrocarbures: par spectrophotométrie en UV et fluorimétrie (Keizer et Gordon, 1973) et par spectroscopie IR (Carlberg et Skarstedt, 1972), qui présentent un intérêt indéniable comme techniques de criblage. Les analyses fines seront effectuées par une combinaison d'analyses chromatographiques haute performance et en phase gazeuse et par chromatographie en phase gazeuse couplée à la spectrométrie de masse (Bouloubassi et Saliot, 1991a; Lipiatou et Saliot, 1991a et références citées). On veillera aux points suivants: la bonne adéquation des conditions opératoires (blancs), l'évaluation des limites de détection et de la reproductibilité du protocole mis en œuvre par des analyses multiples sur le même échantillon, notamment à l'occasion d'exercices d'intercalibration. 


\section{Les hydrocarbures}

\section{dans les sédiments du delta du Rhône et du bassin méditerranéen nord-occidental}

Les investigations conduites récemment sur les hydrocarbures non-aromatiques (HNA) dans les sédiments superficiels donnent des concentrations s'inscrivant dans la gamme de 6,5 à $348,9 \mu \mathrm{g}$ par gramme de sédiment sec, soit de 1,7 à $20,2 \mathrm{mg} / \mathrm{g}$ de carbone organique sédimentaire (CO). Pour les hydrocarbures aromatiques polycycliques (HAP) de masse moléculaire 178, 202, 228, 252 (à l'exception du pérylène) et 276 , la gamme s'étend de 179 à $6363 \mathrm{ng} / \mathrm{g}$, soit de 43,8 à $318 \mu \mathrm{g} / \mathrm{g}$ de $\mathrm{CO}$. On note pour les stations du delta une forte variation des teneurs avec la saison de prélèvement. Les concentrations en HAP varient entre l'été et l'hiver de 1747 à 6363 ng/g à la station Rh1 et de 1070 à 2399 ng/g à la station Rh2, soulignant la sensibilité des sédiments superficiels aux événements polluants, en particulier pyrolytiques plus prononcés en hiver dans les pays de l'hémisphère Nord, par suite de l'accroissement des besoins énergétiques à cette saison. Globalement les teneurs diminuent en fonction de la distance à l'embouchure du Rhône, révélant ainsi la principale source de contamination pour le Golfe du Lion (Lipiatou et Saliot, 1991b). Les sédiments constituent des indicateurs précieux des apports en contaminants, à la condition d'en connaître la vitesse d'accumulation par des mesures isotopiques, et qu'ils soient soumis à une faible bioturbation (Zuo et al., 1991). Ainsi nous avons pu proposer, grâce aux données isotopiques de Zuo et al. (1991), des estimations des flux d'hydrocarbures d'origine pyrolytique et de rétène, un composé d'origine naturelle, issu soit de processus de diagenèse à partir de l'acide abiétique présent dans les résines de conifères, soit de pyrolyse à la suite des feux de forêt (Lipiatou et Saliot, 1991a). Ces résultats sont illustrés sur la figure 3 . On remarquera qu'à la station GL2 le flux d'HAP évalué pour la fin $d u X{ }^{e}$ siècle est inférieur d'un facteur 4 aux valeurs actuelles, alors qu'une tendance contraire est trouvée pour le rétène.

L'intérêt majeur lié à l'analyse fine des hydrocarbures réside dans la détermination de l'origine des différents composés qui peut être naturelle ou anthropique, pétrolière ou pyrolytique. D'autre part, quelque soit la stabilité des hydrocarbures, des transformations se produisent, en particulier dans les zones côtières, transformations physico-chimiques, dues aux forts gradients de salinité, de $\mathrm{pH}$, photo-chimiques et biologiques, en particulier microbiologiques.

Différents critères ont donc été proposés, qui constituent "l'approche multi-diagnostic ": rapport composés résolus/composés non résolus en chromatographie en phase gazeuse, Carbon Preference Index (CPI) des nalcanes traduisant l'importance de la contribution naturelle continentale due aux cires cuticulaires des végétaux supérieurs, abondance des composés isoprénoïdes (pristane et phytane) par rapport aux n-alcanes correspondants 


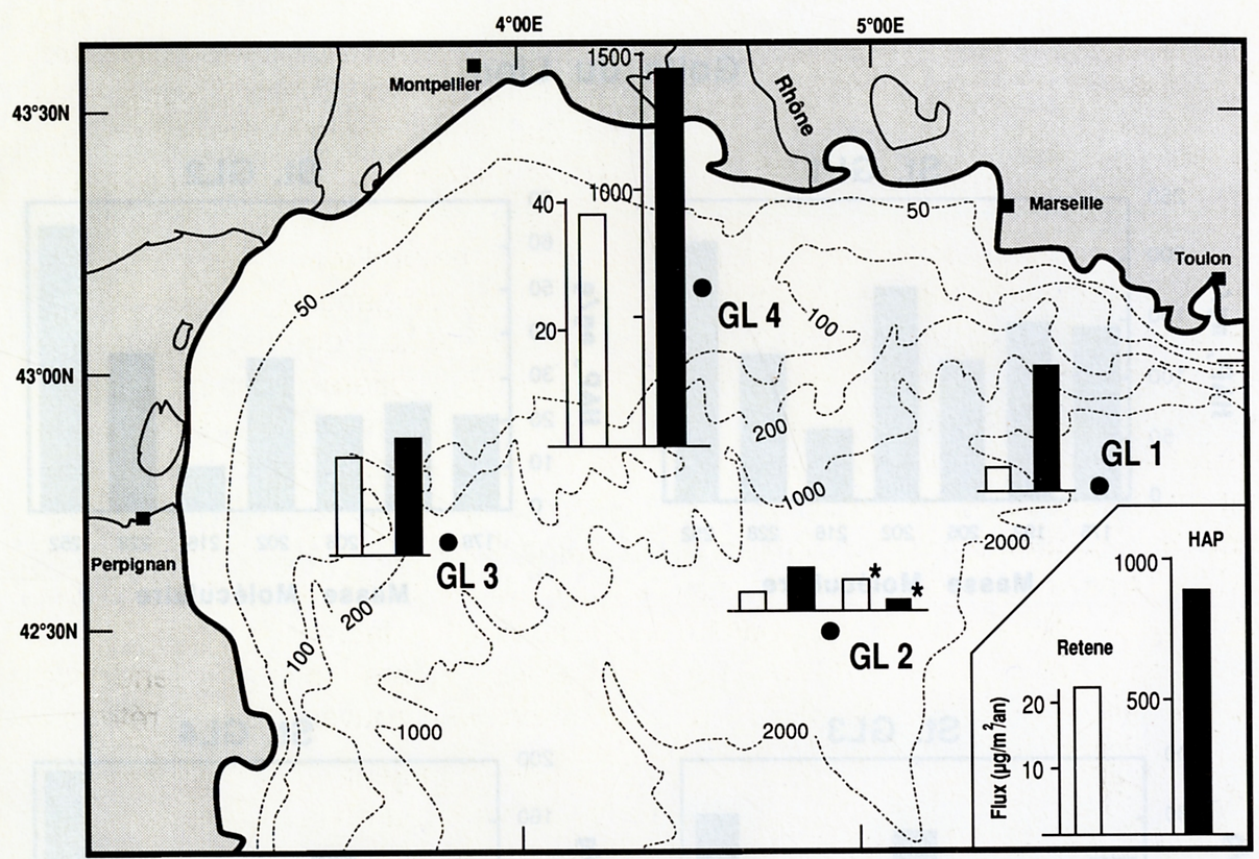

Fig. 3. - Flux annuels des HAP d'origine pyrolytique de masse moléculaire $178,202,228,252$ (-pérylène) et 276 et du rétène enregistrés dans les sédiments superficiels du Golfe du Lion (stations GL1 à GL4). Les flux sont exprimés en $\mu \mathrm{g} / \mathrm{m}^{2} /$ an. Les flux enregistrés à la fin du dix-neuvième siècle sont également donnés pour la station GL2 et notés*. D'après Lipiatou et Saliot (1991a).

Fig. 3. - Annual fluxes of PAH (compounds of molecular mass 178, 202, 228, 252(-perylene) and 276) and retene in surface sediments from the Gulf of Lions (Stations GL1 to GL4). Values are given in $\mu \mathrm{g} / \mathrm{m}^{2} / \mathrm{yr}$. Fluxes corresponding to the end of the nineteenth century are also given at station GL2 (marked*). After Lipiatou and Saliot (1991a).

(n-C17 et $\mathrm{n}-\mathrm{C} 18$ ), type de distribution des $n$-alcanes avec le carbone maximum (Cmax), distribution des hopanes, type de distribution des HAP et présence ou non de composés d'origine pyrolytique anthropique ou de composés d'origine naturelle ou encore de composés d'origine naturelle diagénétique (Albaiges et Albrecht, 1979; Wakeham et al., 1980; Simoneit et Mazurek, 1982; Bouloubassi et Saliot, 1991b; Lipiatou et Saliot, 1991a et références citées).
Nous donnons ci-après deux exemples d'utilisation d'analyses fines de HAP, qui permettent de différencier divers types d'apports en hydrocarbures en milieu marin côtier.

La figure 4, tirée de Lipiatou et Saliot (1991a), montre la distribution d'HAP obtenue à partir de sédiments superficiels prélevés dans le Golfe du Lion (stations GL1, GL2, GL3 et GL4) et dans le delta du Rhône (Rh1 et Rh2). Les concentrations sont repor- 


\section{Golfe du Lion}
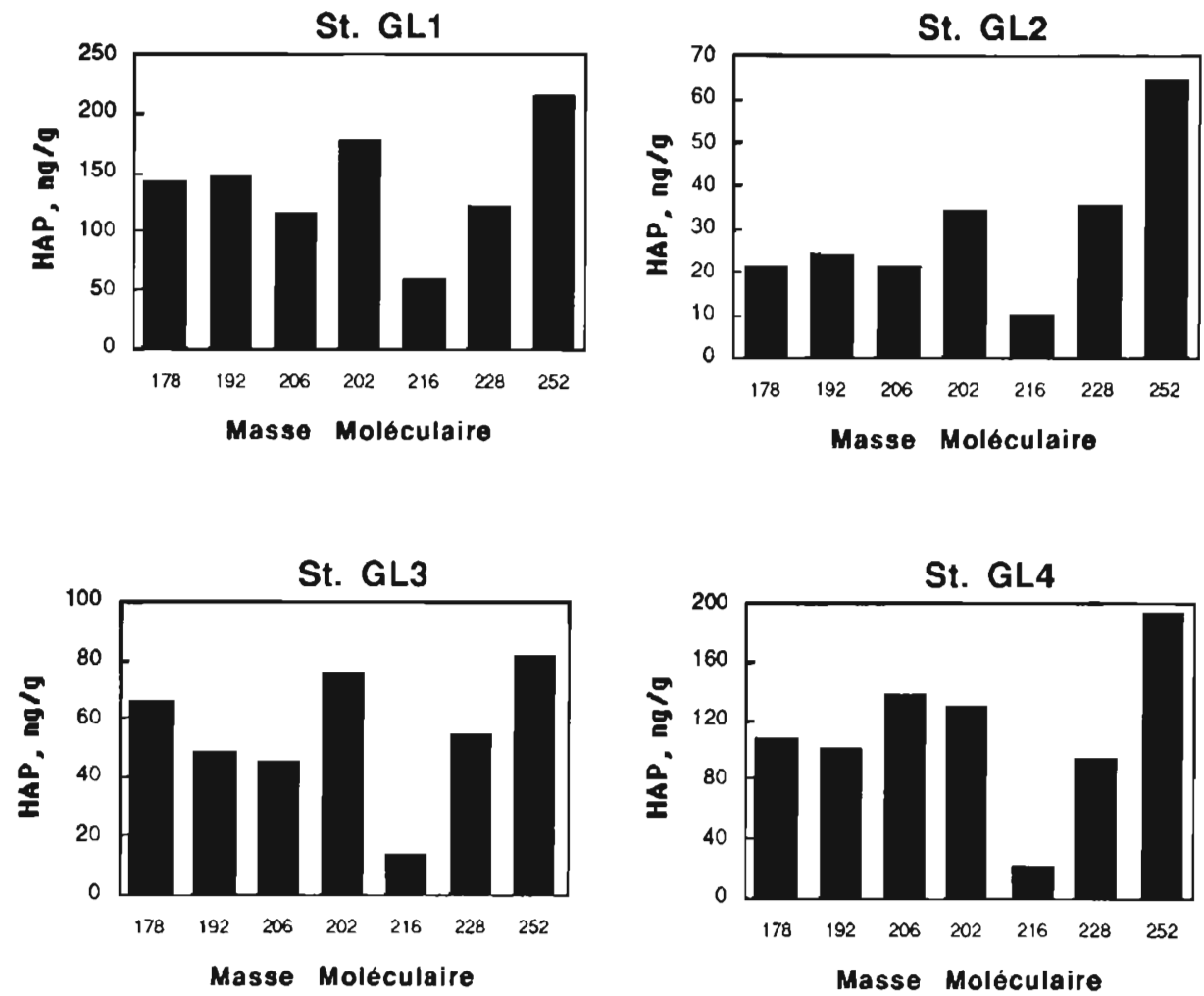

Delta du Rhône
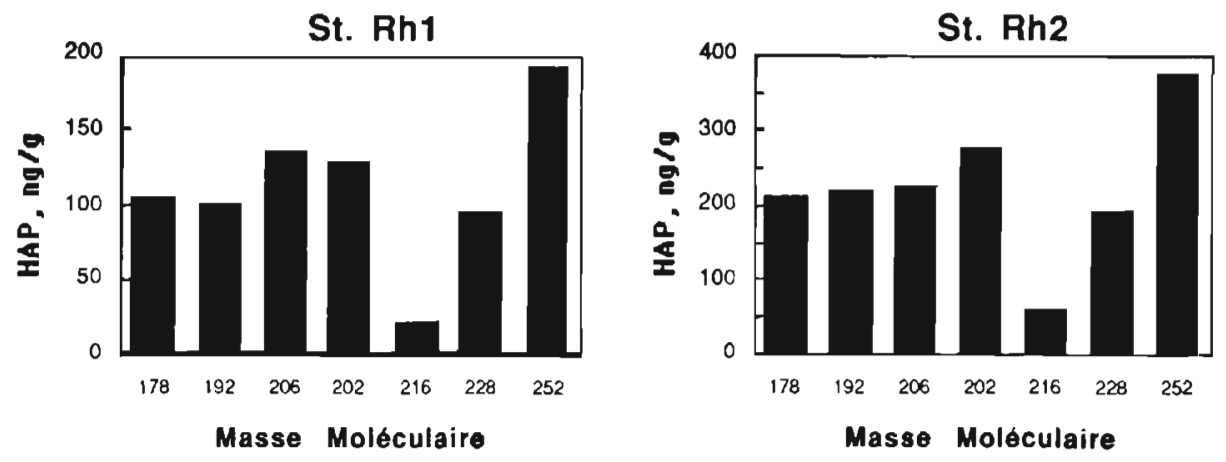
tées en $\mathrm{ng} / \mathrm{g}$ de sédiment sec pour les composés suivants, classés par leur poids moléculaire:

178 : anthracène + phénanthrène;

192: méthyl-anthracènes + méthylphénanthrènes;

206: diméthyl-phénanthrènes;

202: fluoranthène + pyrène;

216: méthyl-pyrènes

228: benzo(a)anthracène + chrysène + triphénylène;

252: benzofluoranthènes + benzo(a) pyrène + benzo(e)pyrène.

La même distribution est observée aussi bien dans les sédiments proches de l'embouchure du Rhône que dans les sédiments du Golfe du Lion prélevés entre $69 \mathrm{~m}$ de fond (GL4) et $2200 \mathrm{~m}$ (GL1), caractérisée par la prédominance des composés de poids moléculaire 202 et 252, reflétant des apports prédominants liés à des processus de pyrolyse à haute température.

La figure 5 tirée de Bouloubassi et Saliot (1991a), montre la distribution du phénanthrène et de ses dérivés substitués, mono-(C1), di-(C2), et tri(C3) méthylés dans des sédiments collectés en été $(E)$ et en hiver $(H)$ devant l'embouchure du Rhône (Rh1) et à la limite du plateau continental, au droit de l'embouchure par 95 mètres de fond (Rh2). Le composé nonsubstitué prédomine sur les homologues substitués. Ceci met en évidence l'origine essentiellement pyrolytique des phénanthrènes. Cependant on ne remarque pas la décroissance nette de l'abondance relative des composés en fonction de leur degré d'alkylation, rencontrée dans les produits de pyrolyse. Au contraire, les diméthyl-phénanthrènes sont légèrement plus abondants que les homologues mono-substitués, révélant également la présence dans ces sédiments d'apports pétrogéniques.

\section{REMERCIEMENTS}

Nous remercions le Dr J.M. Martin, responsable du GRECO CNRS Interactions Continent-Océan et du programme "EROS 2000". Cette recherche a été conduite dans le cadre du programme

Fig. 4. - Distributions des HAP pour divers sédiments superficiels du Golfe du Lion (stations GL1 à GL4) et du delta du Rhône (stations Rh1 et Rh2). Les masses moléculaires, en abscisse, correspondent aux composés suivants: 178: anthracène + phénanthrène; 192: méthyl-anthracènes + mèthyl-phènanthrènes; 206 : diméthyl-phénanthrènes; 202 : fluoranthène + pyrène ; 216 : méthylpyrènes; 228 : benzo(a)anthracène + chrysène + triphénylène: 252 : benzofluoranthènes + benzo (a)pyrène + benzo(e)pyrène. D'après Lipiatou et Saliot (1991a).

Fig. 4 - PAH distribution patterns in sediments from the Gulf of Lions (stations GL1 to GL4) and from the Rhone delta (stations Rh1 and Rh2). Molecular mass of PAH species: 178: anthracene + phenanthrene; 192: methylanthracenes + methyl-phenanthrenes; 206: dimethyl-phenanthrenes; 202: fluoranthene + pyrene; 216: methy\}-pyrenes; 228: benzo(a)anthracene + chrysene + triphenylene; 252: benzofluoranthenes + benzo(a)pyrene + benzo(e)pyrene. After Lipiatou and Saliot (1991a). 


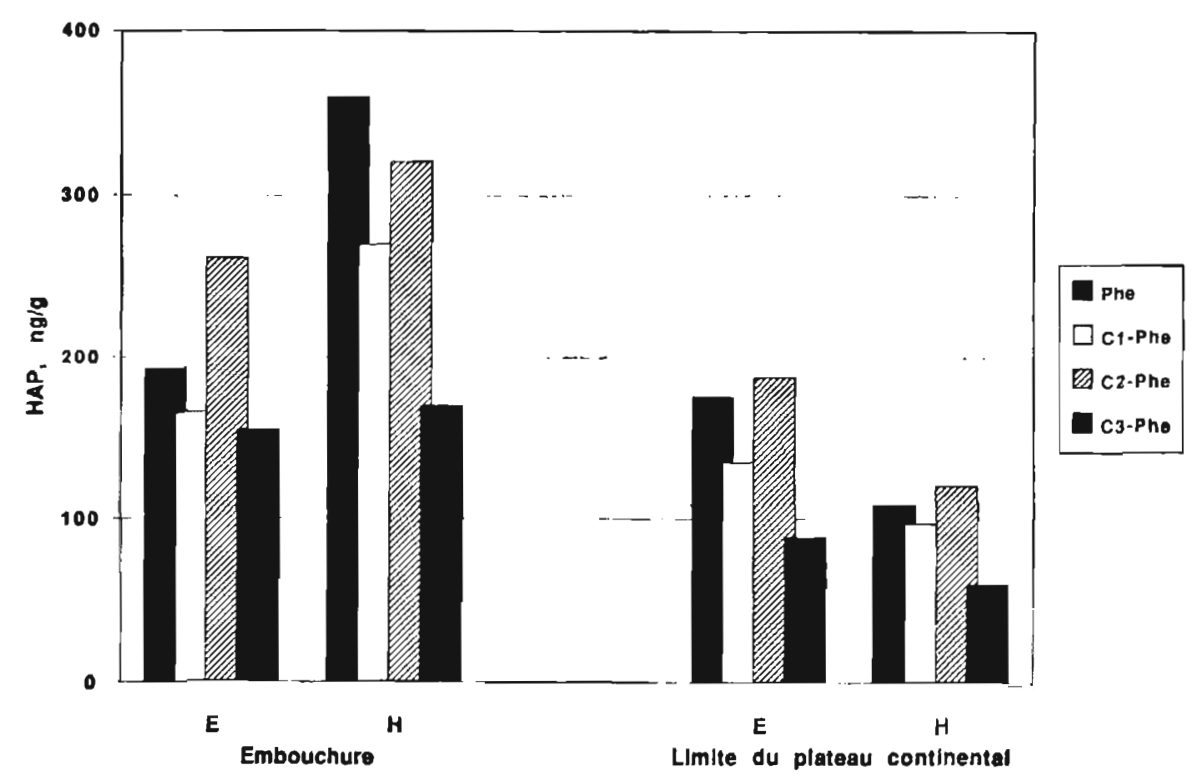

Fig. 5. - Distributions du phénanthrène et de ses homologues alkylés pour des sédiments superficiels prélevés près de l'embouchure du Rhône (Rh1) et à la limite du plateau continental ( $R h 2)$, en été $(E)$ et en hiver $(H)$. C1-, C2- et C3- indiquent les homologues mono-, di- et tri-méthylés, respectivement d'après Bouloubassi et Saliot (1991a).

Fig. 5. - Distribution patterns of phenanthrene and its C1-, C2- and C3- alkylated homologues in superficial sediments from the Rhone delta collected near the river mouth (Rh1) and at the limit of the continental shelf $(\mathrm{Rh} 2)$ in summer $(\mathrm{E})$ and winter $(\mathrm{H})$. After Bouloubassi and Saliot (1991a).

"EROS 2000" (European River Ocean System). Nous remercions l'aide financière du CNRS et de la Commission des Communautés Européennes "4th Environment R \& D Programme "; contrat 4V0111F.

\section{RÉFÉRENCES}

Albaiges J. et Albrecht P., 1979. Fingerprinting marine pollutant hydrocarbons by computerized gas chromatographymass spectrometry. Intern. J. Environ. Anal. Chem., 6, 171-190.

Albaiges J., Grimalt J., Bayona J.M., Risebrough A., Delappe B. et Walker
W.II., 1984. Dissolved, particulate and sedimentary hydrocarbons in a deltaic environment. Org. Geochem., 6, 237248.

Bouloubassi I., 1990. Aspects de la biogéochimie des hydrocarbures non aromatiques et aromatiques dans la colonne d'eau et les sédiments du milieu côtier: cas du delta du Rhône. Thèse de I'Université Pierre et Marie Curie, Paris.

Bouloubassi I. et Saliot A., 1991a. Sources and transport of hydrocarbons in the Rhone delta sediments (Northwestern Mediterranean). Fresenius J. Anal. Chem., 339, 765-771.

Bouloubassi I. et Saliot A., 1991b. Composition and sources of dissolved and particulate PAH in surface waters from 
the Rhone delta (NW Mediterranean). Mar. Pollut. Bull., 22, 588-594.

Burns K.A. et Saliot A., 1986. Petroleum hydrocarbons in the Mediterranean Sea: A mass balance. Mar. Chem., 20, 141-157.

Cariberg S.R. et Skarstedt C.B., 1972. Determination of small amounts of nonpolar hydrocarbons (oil) in sea water. J. Cons. Int. Explor. Mer., 34, 506-515.

Delappe B.W., Risebrough R.W. et Walker W.II., 1983. A large volume sampling assembly for the determination of synthetic organic and petroleum compounds in the dissolved and particulate phases of seawater. Can. J. Fish. Aquat. Sci., 40, 322-336.

Keizer P.D. et Gordon D.C. Jr., 1973. Detection of trace amounts of oil in sea water by fluorescence spectroscopy. $J$. Fish. Res. Board Can., 30, 1039-1046.

Lipiatou E., 1989. Contribution à l'étude géochimique des hydrocarbures aliphatiques et aromatiques dans le delta du Rhône et le Golfe du Lion. Thèse de Doctorat de l'Université Pierre et Marie Curie, Paris.

Lipiatou E. et Saliot A., 1991a. Fluxes and transport of anthropogenic and natural polycyclic aromatic hydrocarbons in the western Mediterranean Sea Mar. Chem., 32, 51-71.

Lipiatou E. et Saliot A., 1991b. Hydrocarbon contamination of the Rhone delta and Western Mediteranean. Mar. Pollut. Bull., 22, 297-304.

Marchand M., 1985. Hydrocarbures et hydrocarbures halogénés dans l'environnement marin. Thèse de Doctorat d'Etat. Université Pierre et Marie Curie, Paris.

NRC, 1985. Oil in the Sea. Inputs, Fates and Effects. National Academic Press, Washington, D.C., $601 \mathrm{pp}$.
Risebrough R.W., Albaiges J. et al., 1983. Application of the mussel watch concept in studies of the distribution of hydrocarbons in the coastal zone. Mar. Pollut. Bull., 4, 181-187.

Saliot A., 1991. Natural hydrocarbons in sea water. In: Marine Organic Chemistry, E.K. Duursma et R. Dawson, eds, Elsevier, Amsterdam, pp. 327-374.

Saliot A. et Marty J.C., 1986. Strategies of sampling and analysis for studying the hydrocarbon pollution at the water-atmosphere interface. In: Strategies and Advanced Techniques for Marine Pollution Studies, $H$. Dou et C.S. Giam, eds, Nato Asi Series, Vol. G9, Springer-Verlag, Berlin, pp. 157186.

Simoneit B.R.T. et Mazurek M.A., 1982. Organic matter in the troposphere- Natural background of biogenic lipid matter in aerosols over the rural Western United States. Atmosph. Environ., 16, 2139-2159.

Span D., 1984. Étude par fractionnement chimique de la distribution des métaux-traces dans les sédiments marins récents: le delta du Rhône, les Golfes de Saint-Raphaël et de Cannes-la-Napoule. Thèse Doct. $3^{e}$ cycle, Université Pierre et Marie Curie, Paris.

Wade S.G. et Quinn J.G., 1975. Hydrocarbons in the Sargasso Sea surface microlayer. Mar. Pollut. Bull., 6, 54-57.

Wakeham S.G., Schaffner C. et Giger W., 1980. Polycyclic aromatic hydrocarbons in Recent lake sediments. I. Compounds having anthropogenic origins. Geochim. Cosmochim. Acta, 44, 403-413.

Zuo Z., Eisma D. et Berger G.W., 1991. Determination of sediment accumulation and mixing rates in the Gulf of Lions, The Mediterranean Sea. Oceanol. Acta, 14, 253-262. 